\title{
CONVOLUTION SAMPLING AND RECONSTRUCTION OF SIGNALS IN A REPRODUCING KERNEL SUBSPACE
}

\author{
M. ZUHAIR NASHED, QIYU SUN, AND JUN XIAN \\ (Communicated by Michael T. Lacey)
}

\begin{abstract}
We consider convolution sampling and reconstruction of signals in certain reproducing kernel subspaces of $L^{p}, 1 \leq p \leq \infty$. We show that signals in those subspaces could be stably reconstructed from their convolution samples taken on a relatively separated set with small gap. Exponential convergence and error estimates are established for the iterative approximationprojection reconstruction algorithm.
\end{abstract}

\section{INTRODUCTION}

In this paper, we consider convolution sampling of signals in a reproducing kernel subspace of $L^{p}:=L^{p}\left(R^{d}\right), 1 \leq p \leq \infty$. Here $L^{p}, 1 \leq p \leq \infty$, is the space of all $p$-integrable functions on the $d$-dimensional Euclidean space with its standard norm denoted by $\|\cdot\|_{p}$.

Convoluting a signal $f \in L^{p}$ with an integrable convolutor $\psi$, the continuous analog of filtering a digital signal, gives

$$
\psi * f:=\int_{\mathbb{R}^{d}} f(y) \psi(\cdot-y) d y .
$$

For a given convolutor $\psi$ and sampling set $\Gamma \subset \mathbb{R}^{d}$, the associated convolution sampling of a signal $f$ yields the data $\{\psi * f(\gamma)\}_{\gamma \in \Gamma}$. This is the ideal sampling of the convoluted signal $\psi * f$ taken on a sampling set $\Gamma$,

$$
f \stackrel{\text { convoluting }}{\longmapsto} \psi * f \stackrel{\text { sampling }}{\longmapsto}\{\psi * f(\gamma)\}_{\gamma \in \Gamma} .
$$

In this paper, a sampling set $\Gamma$ means a relatively separated discrete subset of $\mathbb{R}^{d}$; i.e.,

$$
B_{\Gamma}(\delta):=\sup _{x \in \mathbb{R}^{d}} \sum_{\gamma \in \Gamma} \chi_{[-\delta, \delta]^{d}}(x-\gamma)<\infty \text { for some } \delta>0,
$$

where $\chi_{E}$ is the characteristic function on a set $E[2,4,5]$.

An idempotent operator $T$ on $L^{p}$ is a bounded linear operator $T$ on $L^{p}$ that satisfies

$$
T^{2} f=T f \quad \text { for all } f \in L^{p} \text {. }
$$

Received by the editors September 18, 2011.

2010 Mathematics Subject Classification. Primary 42C15, 41A15, 46A35, 94A12.

Key words and phrases. Convolution sampling, reproducing kernel subspace, iterative algorithm, error estimate. 
The reproducing kernel subspace $V_{p}$ of $L^{p}$ for our signals to live in is the range space of an idempotent integral operator $T$,

$$
V_{p}:=\left\{T f \mid f \in L^{p}\right\},
$$

whose kernel $K$ has certain regularity and decay at infinity [13. Particularly, we assume that the kernel $K$ of the integral operator $T$,

$$
T f(x)=\int_{\mathbb{R}^{d}} K(x, y) f(y) d y, f \in L^{p},
$$

satisfies

$$
\max \left(\sup _{x \in \mathbb{R}^{d}}\|K(x, \cdot)\|_{W^{1}}, \sup _{y \in \mathbb{R}^{d}}\|K(\cdot, y)\|_{W^{1}}\right)<\infty
$$

and

$$
\lim _{\delta \rightarrow 0} \max \left(\sup _{x \in \mathbb{R}^{d}}\left\|\omega_{\delta}(K)(x, \cdot)\right\|_{W^{1}}, \sup _{y \in \mathbb{R}^{d}}\left\|\omega_{\delta}(K)(\cdot, y)\right\|_{W^{1}}\right)=0
$$

Here

$$
W^{1}=\left\{f\left|\|f\|_{W^{1}}:=\left\|\sup _{z \in[-1 / 2,1 / 2)^{d}}|f(\cdot+z)|\right\|_{1}<\infty\right\}\right.
$$

is the Wiener amalgam space, and

$$
\omega_{\delta}(K)(x, y):=\sup _{z_{1}, z_{2} \in[-\delta, \delta]^{d}}\left|K\left(x+z_{1}, y+z_{2}\right)-K(x, y)\right|
$$

is the modulus of continuity of a kernel function $K$ on $\mathbb{R}^{d} \times \mathbb{R}^{d}$.

We recall that the range space $V_{p}$ in (1.3) is a reproducing kernel space [13. Here a closed subspace $V$ of $L^{p}$ is a reproducing kernel subspace of $L^{p}$ such that $\sup _{0 \neq f \in V}|f(x)| /\|f\|_{p}<\infty$ for all $x \in \mathbb{R}^{d}$ [6, 14]. Examples of reproducing kernel subspaces $V_{p}$ of $L^{p}$ include the space of nonuniform splines of order $n$ having $n-$ 1 continuity at each knot [15, 26], the shift-invariant space generated by finitely many functions with certain regularity and decay at infinity [2, 25], and the space modeling signals with finite rate of innovation [7, 20, 23].

In this paper, we study the convolution sampling of signals in the reproducing kernel space $V_{p}$ on a sampling set $\Gamma$ with small gap (Theorems 2.1 and 2.2). We also establish the exponential convergence and error estimates of the iterative approximation-projection algorithm for reconstructing signals from their convolution samples (Theorems 3.1, 4.1 and 4.2).

\section{Stability of CONVOlution SAMPLing}

In this section, we study stability of the convolution sampling for signals in a reproducing kernel subspace of $L^{p}$. We show that any signal in the reproducing kernel subspace $V_{p}$ can be stably reconstructed from its convolution samples taken on a sampling set with small gap, provided that the convolution is stable on $V_{p}$.

To state our main result of this section, we recall the gap of a sampling set and the space of all $p$-summable sequences. A sampling set $\Gamma$ is said to have $\operatorname{gap} \delta>0$ if

$$
A_{\Gamma}(\delta):=\inf _{x \in \mathbb{R}^{d}} \sum_{\gamma \in \Gamma} \chi_{[-\delta, \delta]^{d}}(x-\gamma) \geq 1
$$

([2, 4, 5]). The space of all $p$-summable sequences on a sampling set $\Gamma$ is denoted by $\ell^{p}:=\ell^{p}(\Gamma)$ with its standard norm denoted by $\|\cdot\|_{\ell^{p}(\Gamma)}\left(\right.$ or $\|\cdot\|_{p}$ for short). 
Theorem 2.1. Let $1 \leq p \leq \infty, \psi_{1}, \ldots, \psi_{L}$ be integrable functions on $\mathbb{R}^{d}, V_{p}$ be the reproducing kernel subspace of $L^{p}$ in (1.3) with the kernel $K$ of the associated idempotent operator satisfying (1.4) and (1.5), and set $\Psi=\left(\psi_{1}, \ldots, \psi_{L}\right)^{T}$. Then the following statements are equivalent:

(i) $\Psi$ is a stable convolution sampler on $V_{p}$ for all sampling sets having sufficiently small gap; i.e., there exists $\delta_{0}>0$ such that

$$
0<\inf _{0 \neq f \in V_{p}} \frac{\sum_{l=1}^{L}\left\|\left(\psi_{l} * f(\gamma)\right)_{\gamma \in \Gamma}\right\|_{p}}{\|f\|_{p}} \leq \sup _{\substack{0 \neq f \in V_{p} \\ 0}} \frac{\sum_{l=1}^{L}\left\|\left(\psi_{l} * f(\gamma)\right)_{\gamma \in \Gamma}\right\|_{p}}{\|f\|_{p}}<\infty
$$

holds for any sampling set $\Gamma$ satisfying $1 \leq A_{\Gamma}(\delta) \leq B_{\Gamma}(\delta)<\infty$ for some $\delta \in\left(0, \delta_{0}\right)$.

(ii) $\Psi$ is a stable convolutor on $V_{p}$; i.e.,

$$
0<\inf _{g \in V_{p},\|g\|_{p}=1} \sum_{l=1}^{L}\left\|\psi_{l} * g\right\|_{p} \leq \sup _{g \in V_{p},\|g\|_{p}=1} \sum_{l=1}^{L}\left\|\psi_{l} * g\right\|_{p}<\infty .
$$

We remark that the above equivalence between a stable convolutor on $V_{p}$ and a stable convolution sampler on $V_{p}$ for sampling sets with sufficiently small gap is established in 4. provided that $V_{p}$ is assumed to be a finitely generated shiftinvariant space. The reader may refer to [2, 3, 4, 15, 8, 11, 12, 19, 20, 23, 24, 25, 27, $28,29,30$, and the references therein for sampling and reconstruction of signals in a shift-invariant space and in a reproducing kernel space.

Let $V$ be a closed subspace of $L^{p}$, and $R_{1}, \ldots, R_{L}$ be bounded operators on $L^{p}, 1 \leq p \leq \infty$. We say that $R_{1}, \ldots, R_{L}$ are collectively stable on $V$ if

$$
0<\inf _{g \in V,\|g\|_{p}=1} \sum_{l=1}^{L}\left\|R_{l} g\right\|_{p} \leq \sup _{g \in V,\|g\|_{p}=1} \sum_{l=1}^{L}\left\|R_{l} g\right\|_{p}<\infty
$$

and that $R_{1}, \ldots, R_{L}$ are stable average samplers on $V$ for sampling sets having small gap if there exists a sufficiently small positive number $\delta_{0}$ such that

$$
0<\inf _{g \in V,\|g\|_{p}=1} \sum_{l=1}^{L}\left\|\left(R_{l} g(\gamma)\right)_{\gamma \in \Gamma}\right\|_{p} \leq \sup _{g \in V,\|g\|_{p}=1} \sum_{l=1}^{L}\left\|\left(R_{l} g(\gamma)\right)_{\gamma \in \Gamma}\right\|_{p}<\infty
$$

for any sampling set $\Gamma$ with $1 \leq A_{\Gamma}(\delta) \leq B_{\Gamma}(\delta)<\infty$, where $\delta \in\left(0, \delta_{0}\right)$. In this section, we will prove the following slight generalization of Theorem 2.1 .

Theorem 2.2. Let $1 \leq p \leq \infty, V$ be a closed subspace of $L^{p}$, and let $R_{1}, \ldots, R_{L}$ be integral operators with their kernels $K_{1}, \ldots, K_{L}$ satisfying

$$
\max \left(\sup _{x \in \mathbb{R}^{d}}\left\|K_{l}(x, \cdot)\right\|_{W^{1}}, \sup _{y \in \mathbb{R}^{d}}\left\|K_{l}(\cdot, y)\right\|_{W^{1}}\right)<\infty
$$

and

(2.6) $\lim _{\delta \rightarrow 0} \max \left(\sup _{x \in \mathbb{R}^{d}}\left\|\omega_{\delta}\left(K_{l}\right)(x, \cdot)\right\|_{W^{1}}, \sup _{y \in \mathbb{R}^{d}}\left\|\omega_{\delta}\left(K_{l}\right)(\cdot, y)\right\|_{W^{1}}\right)=0, \quad 1 \leq l \leq L$.

Then integral operators $R_{1}, \ldots, R_{L}$ are collectively stable on $V$ if and only if they are stable average samplers on $V$ for all sampling sets having sufficiently small gap. 
For a moment, we assume that Theorem 2.2 holds and proceed to the proof of Theorem 2.1.

Proof of Theorem 2.1. Given integrable functions $\psi_{1}, \ldots, \psi_{L}$ and a reproducing kernel subspace of $L^{p}$ with the kernel $K$ of the associated idempotent operator satisfying (1.4) and (1.5), we let

$$
K_{l}(x, y)=\int_{\mathbb{R}^{d}} \psi_{l}(z) K(x-z, y) d z
$$

and define

$$
R_{l} f(x)=\int_{\mathbb{R}^{d}} K_{l}(x, y) f(y) d y, \quad 1 \leq l \leq L .
$$

Then for any $1 \leq l \leq L$, it is easy to show that

$$
\left\|R_{l} f\right\|_{p} \leq \max \left(\sup _{x \in \mathbb{R}^{d}}\|K(x, \cdot)\|_{W^{1}}, \sup _{y \in \mathbb{R}^{d}}\|K(\cdot, y)\|_{W^{1}}\right)\left\|\psi_{l}\right\|_{1}\|f\|_{p}
$$

for all $f \in L^{p}$, and hence the integral operator $R_{l}$ is a bounded operator on $L^{p}$. Also for any $1 \leq l \leq L$, the integral operator $R_{l}$ coincides with the convolution operator $\psi_{l}$ on $V_{p}$,

$$
R_{l} f=\psi_{l} * f \quad \text { for all } f \in V_{p},
$$

and its kernel $K_{l}$ satisfies (2.5) and (2.6) . These facts together with Theorem 2.2 lead to the equivalence in Theorem 2.1 .

Now we proceed to prove Theorem 2.2 For a kernel function $K$ on $\mathbb{R}^{d} \times \mathbb{R}^{d}$, define

$$
\|K\|_{\mathcal{W}}:=\max \left(\sup _{x \in \mathbb{R}^{d}}\|K(x, \cdot)\|_{1}, \sup _{y \in \mathbb{R}^{d}}\|K(\cdot, y)\|_{1}\right)<\infty .
$$

The proof of Theorem 2.2 is based on the following two lemmas. The first lemma shows the necessity, while the second one establishes the sufficiency.

Lemma 2.3. Let $1 \leq p \leq \infty, V$ be a closed subspace of $L^{p}$, and let $R_{1}, \ldots, R_{L}$ be integral operators with their kernels $K_{1}, \ldots, K_{L}$ satisfying

$$
\left\|K_{l}\right\|_{\mathcal{W}}<\infty \quad \text { and } \quad \lim _{\delta \rightarrow 0}\left\|\omega_{\delta}\left(K_{l}\right)\right\|_{\mathcal{W}}=0, \quad 1 \leq l \leq L .
$$

If $R_{1}, \ldots, R_{L}$ are collectively stable on $V$, then for any sampling set $\Gamma$ with $1 \leq$ $A_{\Gamma}\left(\delta_{0}\right) \leq B_{\Gamma}\left(\delta_{0}\right)<\infty$ for some $\delta_{0}>0$, we have that

$$
\begin{aligned}
& \left(A_{\gamma}\left(\delta_{0}\right)\right)^{1 / p}\left(\inf _{g \in V,\|g\|_{p}=1} \sum_{l=1}^{L}\left\|R_{l} g\right\|_{p}-\sum_{l=1}^{L}\left\|\omega_{\delta_{0}}\left(K_{l}\right)\right\|_{\mathcal{W}}\right)\|f\|_{p} \\
\leq & \delta_{0}^{1 / p} \sum_{l=1}^{L}\left\|\left(R_{l} f(\gamma)\right)_{\gamma \in \Gamma}\right\|_{p} \\
\leq & \left(B_{\tau}\left(\delta_{0}\right)\right)^{1 / p}\left(\sup _{g \in V,\|g\|_{p}=1} \sum_{l=1}^{L}\left\|R_{l} g\right\|_{p}+\sum_{l=1}^{L}\left\|\omega_{\delta_{0}}\left(K_{l}\right)\right\|_{\mathcal{W}}\right)\|f\|_{p}
\end{aligned}
$$

for all $f \in V$. 
Proof. Let $\delta_{0}>0$ and $\Gamma$ be a sampling set with

$$
1 \leq A_{\Gamma}\left(\delta_{0}\right) \leq B_{\Gamma}\left(\delta_{0}\right)<\infty
$$

Hence $\left\{\gamma+\left[-\delta_{0}, \delta_{0}\right]^{d} \mid \gamma \in \Gamma\right\}$ is a finite covering of $\mathbb{R}^{d}$, and the collection of functions

$$
u_{\gamma}(x)=\frac{\chi_{\left[-\delta_{0}, \delta_{0}\right]^{d}}(x-\gamma)}{\sum_{\gamma^{\prime} \in \Gamma} \chi_{\left[-\delta_{0}, \delta_{0}\right]^{d}}\left(x-\gamma^{\prime}\right)}
$$

defines a bounded uniform partition of unity (BUPU) $U=\left\{u_{\gamma}\right\}_{\gamma \in \Gamma}$; i.e.,

$$
\left\{\begin{array}{l}
0 \leq u_{\gamma}(x) \leq 1 \quad \text { for all } x \in \mathbb{R}^{d} \text { and } \gamma \in \Gamma \\
u_{\gamma}(x)=0 \text { if } x \notin \gamma+\left[-\delta_{0}, \delta_{0}\right]^{d} \\
\sum_{\gamma \in \Gamma} u_{\gamma}(x)=1 \quad \text { for all } x \in \mathbb{R}^{d}
\end{array}\right.
$$

Moreover,

$$
\frac{\delta_{0}^{d}}{B_{\Gamma}\left(\delta_{0}\right)} \leq\left\|u_{\gamma}\right\|_{1} \leq \frac{\delta_{0}^{d}}{A_{\Gamma}\left(\delta_{0}\right)} \quad \text { for all } \gamma \in \Gamma
$$

For all $1 \leq l \leq L$ and $f \in L^{p}, 1 \leq p \leq \infty$, we have that

$$
\left\|\omega_{\delta_{0}}\left(R_{l} f\right)\right\|_{p} \leq\left\|\omega_{\delta_{0}}\left(K_{l}\right)\right\|_{\mathcal{W}}\|f\|_{p}
$$

and

$$
\left|R_{l} f(x)\right|-\omega_{\delta_{0}}\left(R_{l} f\right)(x) \leq R_{l} f(\gamma) \leq\left|R_{l} f(x)\right|+\omega_{\delta_{0}}\left(R_{l} f\right)(x),
$$

where $x \in \gamma+\left[-\delta_{0}, \delta_{0}\right]^{d}, \gamma \in \Gamma$. Then the conclusion (2.12) for $p=\infty$ follows directly from (2.13), 2.17) and (2.18).

For $1 \leq p<\infty$, we obtain from (2.16), (2.17), (2.18) and the stability of $R_{1}, \ldots, R_{L}$ on $V$ that

$$
\begin{aligned}
& \sum_{l=1}^{L}\left\|\left(R_{l} f(\gamma)\right)_{\gamma \in \Gamma}\right\|_{p} \\
\leq & \left(\delta_{0}^{-d} B_{\Gamma}\left(\delta_{0}\right)\right)^{1 / p} \sum_{l=1}^{L}\left(\sum_{\gamma \in \Gamma}\left|R_{l} f(\gamma)\right|^{p}\left\|u_{\gamma}\right\|_{1}\right)^{1 / p} \\
\leq & \left(\delta_{0}^{-d} B_{\Gamma}\left(\delta_{0}\right)\right)^{1 / p} \sum_{l=1}^{L}\left\|\left|R_{l} f\right|+\omega_{\delta_{0}}\left(R_{l} f\right)\right\|_{p} \\
\leq & \left(\delta_{0}^{-d} B_{\Gamma}\left(\delta_{0}\right)\right)^{1 / p}\left(\sum_{l=1}^{L}\left\|R_{l} f\right\|_{p}+\sum_{l=1}^{L}\left\|\omega_{\delta_{0}}\left(K_{l}\right)\right\|_{\mathcal{W}}\|f\|_{p}\right) \\
\leq & \left(\delta_{0}^{-d} B_{\Gamma}\left(\delta_{0}\right)\right)^{1 / p}\left(\sup _{g \in V,\|g\|_{p}=1} \sum_{l=1}^{L}\left\|R_{l} g\right\|_{p}+\sum_{l=1}^{L}\left\|\omega_{\delta_{0}}\left(K_{l}\right)\right\|_{\mathcal{W}}\right)\|f\|_{p}
\end{aligned}
$$


and

$$
\begin{aligned}
& \sum_{l=1}^{L}\left\|\left(R_{l} f(\gamma)\right)_{\gamma \in \Gamma}\right\|_{p} \\
\geq & \left(\delta_{0}^{-d} A_{\Gamma}\left(\delta_{0}\right)\right)^{1 / p} \sum_{l=1}^{L}\left(\sum_{\gamma \in \Gamma}\left|R_{l} f(\gamma)\right|^{p}\left\|u_{\gamma}\right\|_{1}\right)^{1 / p} \\
\geq & \left(\delta_{0}^{-d} A_{\Gamma}\left(\delta_{0}\right)\right)^{1 / p} \sum_{l=1}^{L}\left(\left\|R_{l} f\right\|_{p}-\left\|\omega_{\delta_{0}}\left(R_{l} f\right)\right\|_{p}\right) \\
\geq & \left.\left(\delta_{0}^{-d} A_{\gamma}\left(\delta_{0}\right)\right)^{1 / p} \inf _{g \in V,\|g\|_{p}=1} \sum_{l=1}^{L}\left\|R_{l} g\right\|_{p}-\sum_{l=1}^{L}\left\|\omega_{\delta_{0}}\left(K_{l}\right)\right\|_{\mathcal{W}}\right)\|f\|_{p}
\end{aligned}
$$

for all $1 \leq p<\infty$ and $f \in V$. This proves (2.12) for $1 \leq p<\infty$ and completes the proof.

Lemma 2.4. Let $1 \leq p \leq \infty, V$ be a closed subspace of $L^{p}, R_{1}, \ldots, R_{L}$ be integral operators with their kernels $K_{1}, \ldots, K_{L}$ satisfying (2.5) and (2.6), and let $\Gamma$ be a sampling set with $1 \leq A_{\Gamma}\left(\delta_{0}\right) \leq B_{\Gamma}\left(\delta_{0}\right)<\infty$ for some $\delta_{0}>0$. If

$$
0<\inf _{f \in V,\|f\|_{p}=1} \sum_{l=1}^{L}\left\|\left(R_{l} f(\gamma)\right)_{\gamma \in \Gamma}\right\|_{p} \leq \sup _{f \in V,\|f\|_{p}=1} \sum_{l=1}^{L}\left\|\left(R_{l} f(\gamma)\right)_{\gamma \in \Gamma}\right\|_{p}<\infty
$$

then $R_{1}, \ldots, R_{L}$ are collectively stable on $V$.

Proof. The stability of $R_{1}, \ldots, R_{L}$ for $p=\infty$ follows directly from (2.19) and the assumption on their kernels. Now we assume that $1 \leq p<\infty$. In this case, we have that for $0<\delta \leq \delta_{0}$,

$$
\begin{aligned}
& \sum_{\gamma \in \Gamma} \int_{\gamma+[-\delta / 2, \delta / 2]^{d}}\left|\omega_{\delta}\left(R_{l} f\right)(x)\right|^{p} d x \\
\leq & \sum_{\gamma \in \Gamma} \int_{\gamma+[-\delta / 2, \delta / 2]^{d}}\left|\int_{\mathbb{R}^{d}} \omega_{\delta}\left(K_{l}\right)(x, y)\right| f(y)|d y|^{p} d x \\
\leq & \left(\sup _{x \in \mathbb{R}^{d}}\left\|\omega_{\delta}\left(K_{l}\right)(x, \cdot)\right\|_{1}\right)^{p-1} \sum_{\gamma \in \Gamma} \int_{\gamma+[-\delta / 2, \delta / 2]^{d}} \int_{\mathbb{R}^{d}} \omega_{\delta}\left(K_{l}\right)(x, y)|f(y)|^{p} d y d x \\
\leq & \left(\sup _{y \in \mathbb{R}^{d}} \sum_{k \in \mathbb{Z}^{d}} \sum_{\gamma \in \Gamma} \int_{\left(\gamma+[-\delta / 2, \delta / 2]^{d}\right) \cap\left(k+[-1 / 2,1 / 2]^{d}\right)} \omega_{\delta}\left(K_{l}\right)(x, y) d x\right) \\
& \times\left(\sup _{x \in \mathbb{R}^{d}}\left\|\omega_{\delta}\left(K_{l}\right)(x, \cdot)\right\|_{W^{1}}\right)^{p-1}\|f\|_{p}^{p} \\
\leq & \left(\sup _{z \in \mathbb{R}^{d}} \sum_{\gamma \in \Gamma}\left|\left(\gamma+[-\delta / 2, \delta / 2]^{d}\right) \cap\left(z+[-1 / 2,1 / 2]^{d}\right)\right|\right) \\
& \times\left(\sup _{x \in \mathbb{R}^{d}}\left\|\omega_{\delta}\left(K_{l}\right)(x, \cdot)\right\|_{W^{1}}\right)^{p-1}\left(\sup _{y \in \mathbb{R}^{d}}\left\|\omega_{\delta}\left(K_{l}\right)(\cdot, y)\right\|_{W^{1}}\right)\|f\|_{p}^{p} \\
\leq & B_{\Gamma}\left(1+\delta_{0}\right)\left(\sup _{x \in \mathbb{R}^{d}}\left\|\omega_{\delta}\left(K_{l}\right)(x, \cdot)\right\|_{W^{1}}\right)^{p-1}\left(\sup _{y \in \mathbb{R}^{d}}\left\|\omega_{\delta}\left(K_{l}\right)(\cdot, y)\right\|_{W^{1}}\right) \delta^{d}\|f\|_{p}^{p} .
\end{aligned}
$$


This implies that

$$
\begin{aligned}
& \left(B_{\Gamma}\left(\delta_{0}\right)\right)^{1 / p} \sum_{l=1}^{L}\left\|R_{l} f\right\|_{p} \\
\geq & \sum_{l=1}^{L}\left(\sum_{\gamma \in \Gamma} \int_{\gamma+[-\delta / 2, \delta / 2]^{d}}\left|R_{l} f(x)\right|^{p} d x\right)^{1 / p} \\
\geq & \delta^{d / p} \sum_{l=1}^{L}\left(\sum_{\gamma \in \Gamma}\left|R_{l} f(\gamma)\right|^{p}\right)^{1 / p}-\sum_{l=1}^{L}\left(\sum_{\gamma \in \Gamma} \int_{\gamma+[-\delta / 2, \delta / 2]^{d}}\left|\omega_{\delta}\left(R_{l} f\right)(x)\right|^{p} d x\right)^{1 / p} \\
\geq & \delta^{d / p}\|f\|_{p}\left(\inf _{g \in V,\|g\|_{p}=1} \sum_{l=1}^{L}\left\|\left(R_{l} g(\gamma)\right)_{\gamma \in \Gamma}\right\|_{p}-\left(B_{\Gamma}\left(1+\delta_{0}\right)\right)^{1 / p}\right. \\
& \times\left(\sum_{l=1}^{L}\left(\sup _{x \in \mathbb{R}^{d}}\left\|\omega_{\delta}\left(K_{l}\right)(x, \cdot)\right\|_{W^{1}}\right)^{(p-1) / p}\left(\sup _{y \in \mathbb{R}^{d}}\left\|\omega_{\delta}\left(K_{l}\right)(\cdot, y)\right\|_{W^{1}}\right)^{1 / p}\right) \\
\geq & \frac{1}{2} \delta^{d / p}\left(\inf _{g \in V,\|g\|_{p}=1} \sum_{l=1}^{L} \|\left(R_{l} g(\gamma)\right)_{\left.\gamma \in \Gamma \|_{p}\right)\|f\|_{p}}\right.
\end{aligned}
$$

by (2.6), when $\delta$ is chosen sufficiently small. Hence the stability of the integral operators $R_{1}, \ldots, R_{L}$ on $V$ for $1 \leq p<\infty$ follows.

Finally we prove Theorem 2.2 .

Proof of Theorem 2.2. The necessity follows from Lemma 2.3, while the sufficiency holds by Lemma 2.4

\section{Exponential CONVERgence of the iterative APPROXIMATION-PROJECTION RECONSTRUCTION ALGORITHM}

Let $\mathcal{B}\left(L^{p}\right), 1 \leq p \leq \infty$, be the Banach algebra of bounded linear operators on $L^{p}$ and denote by $\|\cdot\|_{\mathcal{B}\left(L^{p}\right)}$ its norm. In this section, we establish exponential convergence of the iterative approximation-projection algorithm to reconstruct signals in a reproducing kernel subspace $V_{p}$ of $L^{p}, 1 \leq p \leq \infty$, from their convolution samples on a sampling set with small gap.

Theorem 3.1. Let $1 \leq p \leq \infty$ and $V$ be a closed subspace of $L^{p}$. Assume that integral operators $R_{1}, \ldots, R_{L}$ are stable convolutors on $V$ whose kernels $K_{1}, \ldots, K_{L}$ satisfy (2.5) and (2.6) and that $\tilde{R}_{1}, \ldots, \tilde{R}_{L}$ are bounded operators from $L^{p}$ to $V$ satisfying

$$
\sum_{l=1}^{L} \tilde{R}_{l}\left(R_{l} f\right)=f \quad \text { for all } f \in V .
$$

Given a sample set $\Gamma$ with $1 \leq A_{\Gamma}\left(\delta_{0}\right) \leq B_{\Gamma}\left(\delta_{0}\right)<\infty$ for some $\delta_{0}>0$, let $\left\{u_{\gamma}\right\}_{\gamma \in \Gamma}$ be a bounded uniform partition of unity in (2.15) and define an operator $P$ from $L^{p}$ to $V$ by

$$
P f(x)=\sum_{l=1}^{L} \sum_{\gamma \in \Gamma} R_{l} f(\gamma) \tilde{R}_{l} u_{\gamma}(x), \quad f \in L^{p} .
$$


Then given samples $R_{l} g(\gamma), \gamma \in \Gamma$, of a signal $g \in V$, the iterative algorithm

$$
g_{0}=P g \quad \text { and } \quad g_{n}=g_{0}+g_{n-1}-P g_{n-1} \text { when } n \geq 1
$$

converges exponentially to $g \in V$, provided that

$$
r:=\sum_{l=1}^{L}\left\|\tilde{R}_{l}\right\|_{\mathcal{B}\left(L^{p}\right)}\left\|\omega_{\delta_{0}}\left(K_{l}\right)\right\|_{\mathcal{W}}<1 .
$$

Moreover

$$
\left\|g_{n}-g\right\|_{p} \leq \frac{r^{n+1}}{1-r}\|P g\|_{p} \quad \text { for all } g \in V .
$$

Let $T$ be an integral idempotent operator whose kernel satisfies (1.4) and (1.5) and let $V_{p}$ be the corresponding reproducing kernel subspace of $L^{p}$ in (1.3). Using an argument similar to the one in the proof of Theorem 2.1 and applying Theorem 3.1 with $R_{l} f$ replaced by $\psi_{l} * T f, 1 \leq l \leq L$, we obtain the exponential convergence of the iterative approximation-projection algorithm for reconstructing signals in a reproducing kernel subspace $V_{p}$ of $L^{p}$ from their convolution samples.

Corollary 3.2. Let $1 \leq p \leq \infty, T$ be an integral idempotent operator whose kernel satisfies (1.4) and (1.5), and $V_{p}$ be the corresponding reproducing kernel subspace of $L^{p}$ in (1.3). Assume that $\Psi:=\left(\psi_{1}, \ldots, \psi_{L}\right)$ is a stable convolutor on $V_{p}$ and has its components $\psi_{1}, \ldots, \psi_{L}$ being integrable, and that $\tilde{R}_{1}, \ldots, \tilde{R}_{L}$ are bounded operators from $L^{p}$ to $V_{p}$ satisfying $\sum_{l=1}^{L} \tilde{R}_{l}\left(\psi_{l} * f\right)=f$ for all $f \in V_{p}$. Given a sample set $\Gamma$ with $1 \leq A_{\Gamma}\left(\delta_{0}\right) \leq B_{\Gamma}\left(\delta_{0}\right)<\infty$ for some $\delta_{0}>0$, let $\left\{u_{\gamma}\right\}_{\gamma \in \Gamma}$ be a bounded uniform partition of unity in (2.15) and define an operator $Q$ from $L^{p}$ to $V_{p}$ by

$$
Q f(x)=\sum_{l=1}^{L} \sum_{\gamma \in \Gamma} \psi_{l} * f(\gamma) \tilde{R}_{l} u_{\gamma}(x), \quad f \in L^{p} .
$$

Then given convolution samples $\psi_{l} * g(\gamma), \gamma \in \Gamma, 1 \leq l \leq L$, of a signal $g \in V_{p}$, the iterative approximation-projection algorithm

$$
g_{0}=Q g \quad \text { and } \quad g_{n}=g_{0}+g_{n-1}-Q g_{n-1} \text { when } n \geq 1
$$

converges exponentially to $g \in V$, provided that

$$
r:=\left(\sum_{l=1}^{L}\left\|\tilde{R}_{l}\right\|_{\mathcal{B}\left(L^{p}\right)}\left\|\psi_{l}\right\|_{1}\right)\left\|\omega_{\delta_{0}}(K)\right\|_{\mathcal{W}}<1 .
$$

Moreover $\left\|g_{n}-g\right\|_{p} \leq \frac{r^{n+1}}{1-r}\|Q g\|_{p}$ for all $g \in V_{p}$.

Remark 3.1. The iterative algorithm (3.3) can be thought of as a generalization of the approximation-projection reconstruction algorithm in [9] for convolution sampling. We remark that the approximation-projection algorithm was originally introduced in 9 for reconstructing band-limited signals and was later generalized in [1] to the recovery of signals in a shift-invariant space; see [2, 4, 5, 13] and the references therein for various generalizations and applications of that reconstruction algorithm. 
Remark 3.2. The bounded operators $\tilde{R}_{1}, \ldots, \tilde{R}_{l}$ in (3.1) can be interpreted as the inverse of integral operators $R_{1}, \ldots, R_{L}$ on the closed subspace $V$. The existence problem of such bounded operators is open, except that $V$ is a finitely generated shift-invariant space and $R_{1}, \ldots, R_{L}$ are convolution operators. As the integral operators $R_{1}, \ldots, R_{l}$ have their kernels with certain regularity and decay at infinity, we expect that $\tilde{R}_{1}, \ldots, \tilde{R}_{L}$ can be chosen to have the same property. This is a very interesting topic closely related to Wiener's lemma for localized integral operators. The reader may refer to [10, 16, 18, 21, 22, and the references therein for Wiener's lemma for infinite matrices and localized integral operators.

Proof of Theorem 3.1. The operator $P$ in (3.2) is well defined because $\tilde{R}_{l}, 1 \leq l \leq$ $L$, are bounded operators on $L^{p}$ by the assumption, and $\sum_{\gamma \in \Gamma} R_{l} f(\gamma) u_{\gamma}, 1 \leq l \leq L$, belongs to $L^{p}$ as $\left(R_{l} f(\gamma)\right)_{\gamma \in \Gamma} \in \ell^{p}(\Gamma)$ by Lemma 2.3. Let $f \in V \subset L^{p}, 1 \leq p \leq \infty$. Then for $1 \leq p<\infty$,

$$
\begin{aligned}
\|f-P f\|_{p}= & \left(\int_{\mathbb{R}^{d}}\left|f(x)-\sum_{l=1}^{L} \sum_{\gamma \in \Gamma} R_{l} f(\gamma) \tilde{R}_{l} u_{\gamma}(x)\right|^{p} d x\right)^{1 / p} \\
= & \left(\int_{\mathbb{R}^{d}}\left|\sum_{l=1}^{L} \tilde{R}_{l}\left(R_{l} f-\sum_{\gamma \in \Gamma} R_{l} f(\gamma) u_{\gamma}\right)(x)\right|^{p} d x\right)^{1 / p} \\
\leq & \sum_{l=1}^{L}\left\|\tilde{R}_{l}\right\|_{\mathcal{B}\left(L^{p}\right)} \\
& \times\left(\int_{\mathbb{R}^{d}}\left|\int_{\mathbb{R}^{d}}\left(K_{l}(x, y)-\sum_{\gamma \in \Gamma} K_{l}(\gamma, y) u_{\gamma}(x)\right) f(y) d y\right|^{p} d x\right)^{1 / p} \\
\leq & \left(\sum_{l=1}^{L}\left\|\tilde{R}_{l}\right\|_{\mathcal{B}\left(L^{p}\right)}\left\|\omega_{\delta_{0}}\left(K_{l}\right)\right\|_{\mathcal{W}}\right)\|f\|_{p},
\end{aligned}
$$

and for $p=\infty$,

$$
\begin{aligned}
& \|f-P f\|_{p}=\sup _{x \in \mathbb{R}^{d}}\left|f(x)-\sum_{l=1}^{L} \sum_{\gamma \in \Gamma} R_{l} f(\gamma) \tilde{R}_{l} u_{\gamma}(x)\right| \\
\leq & \sum_{l=1}^{L}\left\|\tilde{R}_{l}\right\|_{\mathcal{B}\left(L^{\infty}\right)} \sup _{x \in \mathbb{R}^{d}}\left|\int_{\mathbb{R}^{d}}\left(K_{l}(x, y)-\sum_{\gamma \in \Gamma} K_{l}(\gamma, y) u_{\gamma}(x)\right) f(y) d y\right| \\
\leq & \left(\sum_{l=1}^{L}\left\|\tilde{R}_{l}\right\|_{\mathcal{B}\left(L^{\infty}\right)}\left\|\omega_{\delta_{0}}\left(K_{l}\right)\right\| \mathcal{W}\right)\|f\|_{\infty} .
\end{aligned}
$$

From (3.3) it follows that

$$
f_{n+1}-f_{n}=(I-P)\left(f_{n}-f_{n-1}\right)=\cdots=(I-P)^{n+1} P f
$$

for all $n \geq 0$. This together with (3.4), (3.8) and (3.9) proves (3.5), and hence the exponential convergence of $f_{n}, n \geq 0$, defined in (3.3). 


\section{Error estimates of the iterative approximation-Projection RECONSTRUCTION ALGORITHM}

In this section, we first consider the iterative approximation-projection algorithm (3.3) when samples are corrupted, that is $\left\{R_{l} g(\gamma)+\epsilon_{l}(\gamma)\right\}$, where $\left\{\epsilon_{l}(\gamma)\right\}$ is noise.

Theorem 4.1. Let $1 \leq p \leq \infty, V$ be a closed subspace of $L^{p}$, integral operators $R_{1}, \ldots, R_{L}$ be stable convolutors on $V$ whose kernels $K_{1}, \ldots, K_{L}$ satisfy (2.5) and (2.6), and $\tilde{R}_{1}, \ldots, \tilde{R}_{L}$ be bounded operators from $L^{p}$ to $V$ satisfying (3.1). Given a sample set $\Gamma$ with $1 \leq A_{\Gamma}\left(\delta_{0}\right) \leq B_{\Gamma}\left(\delta_{0}\right)<\infty$ for some $\delta_{0}>0$, let $\left\{u_{\gamma}\right\}_{\gamma \in \Gamma}$ be a bounded uniform partition of unity in (2.15) and define an operator $P$ from $L^{p}$ to $V$ as in (3.2). Then given noisy samples $R_{l} g(\gamma)+\epsilon_{l}(\gamma), \gamma \in \Gamma$ of a signal $g \in V$ with $\epsilon_{l}:=\left(\epsilon_{l}(\gamma)\right)_{\gamma \in \Gamma} \in \ell^{p}(\Gamma)$, the iterative approximation-projection algorithm

$$
\left\{\begin{array}{l}
\tilde{g}_{0}=\sum_{l=1}^{L} \sum_{\gamma \in \Gamma}\left(R_{l} g(\gamma)+\epsilon_{l}(\gamma)\right) \tilde{R}_{l} u_{\gamma} \\
\tilde{g}_{n}=\tilde{g}_{0}+\tilde{g}_{n-1}-P \tilde{g}_{n-1} \text { when } n \geq 1
\end{array}\right.
$$

converges exponentially to $\tilde{g} \in V$ provided that (3.4) holds. Moreover

$$
\|g-\tilde{g}\|_{p} \leq \frac{\delta_{0}^{d / p} \sum_{l=1}^{L}\left\|\tilde{R}_{l}\right\|_{\mathcal{B}\left(L^{p}\right)}\left\|\left(\epsilon_{l}(\gamma)\right)_{\gamma \in \Gamma}\right\|_{p}}{\left(A_{\Gamma}\left(\delta_{0}\right)\right)^{1 / p}\left(1-\sum_{l=1}^{L}\left\|\tilde{R}_{l}\right\|_{\mathcal{B}\left(L^{p}\right)}\left\|\omega_{\delta_{0}}\left(K_{l}\right)\right\|_{\mathcal{W})}\right.}
$$

Proof. Set $h_{0}=\sum_{l=1}^{L} \sum_{\gamma \in \Gamma} \epsilon_{l}(\gamma) \tilde{R}_{l} u_{\gamma}$, and define $g_{n}, n \geq 0$, by

$$
g_{0}=P g \quad \text { and } \quad g_{n}=g_{0}+g_{n-1}-P g_{n-1} \text { when } n \geq 1
$$

Then $h_{0} \in V$ and

$$
\tilde{g}_{n}-g_{n}=h_{0}+(I-P) h_{0}+\cdots+(I-P)^{n} h_{0}, n \geq 0 .
$$

This together with (3.8), (3.9) and Theorem 3.1 leads to the exponential convergence of the sequence $\tilde{g}_{n}, n \geq 0$, to a function $\tilde{g} \in V$. Moreover,

$$
\begin{aligned}
\|\tilde{g}-g\|_{p} & \leq \sum_{n=0}^{\infty}\left\|(I-P)^{n} h_{0}\right\|_{p} \leq \frac{\left\|h_{0}\right\|_{p}}{1-r} \\
& \leq(1-r)^{-1} \sum_{l=1}^{L}\left\|\tilde{R}_{l}\right\|_{\mathcal{B}\left(L^{p}\right)}\left\|\sum_{\gamma \in \Gamma} \epsilon_{l}(\gamma) u_{\gamma}\right\|_{p} \\
& \leq(1-r)^{-1} \sum_{l=1}^{L}\left\|\tilde{R}_{l}\right\|_{\mathcal{B}\left(L^{p}\right)}\left\|\left(\epsilon_{l}(\gamma)\right)_{\gamma \in \Gamma}\right\|_{p}\left(\sup _{\gamma \in \Gamma}\left\|u_{\gamma}\right\|_{1}\right)^{1 / p} .
\end{aligned}
$$

This together with (2.16) proves (4.1).

We conclude this section by considering the iterative approximation-projection reconstruction algorithm (3.3) when the iterative algorithm is corrupted, that is, when each iteration step is corrupted by noise $\left\{h_{n}\right\}$. 
Theorem 4.2. Let $1 \leq p \leq \infty, V$ be a closed subspace of $L^{p}$, integral operators $R_{1}, \ldots, R_{L}$ be stable convolutors on $V$ whose kernels $K_{1}, \ldots, K_{L}$ satisfy (2.5) and (2.6), and $\tilde{R}_{1}, \ldots, \tilde{R}_{L}$ be bounded operators from $L^{p}$ to $V$ satisfying (3.1). Given a sample set $\Gamma$ with $1 \leq A_{\Gamma}\left(\delta_{0}\right) \leq B_{\Gamma}\left(\delta_{0}\right)<\infty$ for some $\delta_{0}>0$, let $\left\{u_{\gamma}\right\}_{\gamma \in \Gamma}$ be a bounded uniform partition of unity in (2.15) and define an operator $P$ from $L^{p}$ to $V$ as in (3.2). Given samples $R_{l} g(\gamma), \gamma \in \Gamma$, of a signal $g \in V$, assume that the iterative approximation-projection algorithm

$$
\tilde{g}_{0}=P g+h_{0} \quad \text { and } \quad \tilde{g}_{n}=\tilde{g}_{0}+\tilde{g}_{n-1}-P \tilde{g}_{n-1}+h_{n} \text { when } n \geq 1
$$

is corrupted by $h_{n} \in V, n \geq 0$, during the implementation. Then

$$
\left\|\tilde{g}_{n}-g\right\|_{p} \leq \frac{r^{n}}{1-r}\|P g\|_{p}+\frac{1}{1-r}\left\|h_{0}\right\|_{p}+\sum_{l=1}^{n} r^{n-l}\left\|h_{l}\right\|_{p}, \quad n \geq 1,
$$

provided that (3.4) holds, where $r=\sum_{l=1}^{L}\left\|\tilde{R}_{l}\right\|_{\mathcal{B}\left(L^{p}\right)}\left\|\omega_{\delta_{0}}\left(K_{l}\right)\right\|_{\mathcal{W}} \in(0,1)$.

Proof. Let $g_{n}, n \geq 0$, be as in (4.1). Then

$$
\tilde{g}_{n}=g_{n}+\sum_{l=0}^{n}(I-P)^{l} h_{0}+\sum_{l=1}^{n}(I-P)^{n-l} h_{l}, n \geq 1 .
$$

Then (4.4) follows from (3.8), (3.9), (3.10) and (4.5).

Remark 4.1. We remark that $h_{n}, n \geq 0$, in the implementation (4.3) is assumed to belong to a subspace $V$ of $L^{p}$ instead of living in the whole space $L^{p}$ with more freedom, as we notice that in that general case the error in the implementation could accumulate quickly at each step; cf. [13. On the other hand, the assumption that $h_{n} \in V, n \geq 0$, can be reached easily by replacing $h_{n} \in L^{p}$ by $\tilde{h}_{n}:=\sum_{l=1}^{L} \tilde{R}_{l}\left(R_{l} h_{n}\right) \in V$.

\section{ACKNOWLEDGEMENTS}

The authors wish to thank the anonymous referees for their valuable suggestions. The second author is partially supported by the National Science Foundation (DMS1109063). The third author is the corresponding author and is partially supported by the National Natural Science Foundation of China (10801136, 10871213), the Scientific Research Foundation for the Returned Overseas Chinese Scholars, State Education Ministry, the Fundamental Research Funds for the Central Universities (10lgpy27) and Guangdong Provincial Government of China through the Computational Science Innovative Research Team program and Guangdong Province Key Laboratory of Computational Science at the Sun Yat-sen University.

\section{REFERENCES}

[1] A. Aldroubi and H. Feichtinger, Exact iterative reconstruction algorithm for multivariate irregularly sampled functions in spline-like spaces: the $L^{p}$ theory, Proc. Amer. Math. Soc., 126(1998), 2677-2686. MR1451788 (99h:42054)

[2] A. Aldroubi and K. Gröchenig, Nonuniform sampling and reconstruction in shift-invariant space, SIAM Review, 43(2001), 585-620. MR1882684(2003e:94040)

[3] A. Aldroubi, C. Leonetti and Q. Sun, Error analysis of frame reconstruction from noisy samples, IEEE Transactions on Signal Processing, 56(2008), 2311-2325. MR.2516635 
[4] A. Aldroubi, Q. Sun and W.-S. Tang, Convolution, average sampling, and a Calderon resolution of the identity for shift-invariant spaces, J. Fourier Anal. Appl., 11(2005), 215-244. MR2131637 (2006d:42048)

[5] A. Aldroubi, Q. Sun and W.-S. Tang, Nonuniform average sampling and reconstruction in multiply generated shift-invariant spaces, Constr. Approx., 20(2004), 173-189. MR2036639 (2005d:42036)

[6] N. Aronszajn, Theory of reproducing kernels, Trans. Amer. Math. Soc., 68(1950), 337-404. $\operatorname{MR} 0051437(14: 479 \mathrm{c})$

[7] N. Bi, M. Z. Nashed and Q. Sun, Reconstructing signals with finite rate of innovation from noisy samples, Acta Appl. Math., 107(2009), 339-372. MR2520024(2010e:94114)

[8] J. G. Christensen, Sampling in reproducing kernel Banach spaces on Lie groups, J. Approx. Theory, 164(2012), 179-203. MR2855776 (2012k:42061)

[9] H. G. Feichtinger and K. Gröchenig, Iterative reconstruction of multivariate bandlimited functions from irregular sampling values, SIAM J. Math. Anal., 23(1992), 244-261. MR.1145171 (93a:94006)

[10] K. Gröchenig, Wiener's lemma: Theme and variations, an introduction to spectral invariance and its applications, In Four Short Courses on Harmonic Analysis: Wavelets, Frames, TimeFrequency Methods, and Applications to Signal and Image Analysis, edited by P. Massopust and B. Forster, Birkhäuser, Boston, 2010. MR2640516 (2011e:42002)

[11] D. Han, M. Z. Nashed and Q. Sun, Sampling expansions in reproducing kernel Hilbert and Banach spaces, Numer. Funct. Anal. Optim., 30(2009), 971-987. MR2589760(2010m:42062)

[12] Y. Liu and G. G. Walter, Irregular sampling in wavelet subspaces, J. Fourier Anal. Appl., 2(1995), 181-189. MR1365204 (96k:42033)

[13] M. Z. Nashed and Q. Sun, Sampling and reconstruction of signals in a reproducing kernel subspace of $L^{p}\left(R^{d}\right)$, J. Funct. Anal., 258(2010), 2422-2452. MR2584749 (2011a:60160)

[14] M. Z. Nashed and G. G. Walter, General sampling theorems for functions in reproducing kernel Hilbert spaces, Math. Control Signals Systems, 4(1991), 363-390. MR.1128261 (92h:41063)

[15] L. L. Schumaker, Spline functions: basic theory, John Wiley \& Sons, New York (1981). MR606200(82j:41001)

[16] C. E. Shin and Q. Sun, Stability of localized operators, J. Funct. Anal., 256(2009), 24172439. MR 2502521(2010e:47012)

[17] S. Smale, D. X. Zhou. Shannon sampling. II: Connections to learning theory, Appl. Comput. Harmon. Anal., 19(3)(2005), 285-302. MR2186447 (2006i:94024)

[18] Q. Sun, Wiener's lemma for infinite matrices II, Constr. Approx., 34(2011), 209-235. MR2822769

[19] Q. Sun, Local reconstruction for sampling in shift-invariant spaces, Adv. Comput. Math., 32(2010), 335-352. MR2595176 (2011a:94045)

[20] Q. Sun, Frames in spaces with finite rate of innovation, Adv. Comp. Math., 28(2008), 301329. MR2390281 (2009c:42093)

[21] Q. Sun, Wiener's lemma for localized integral operators, Appl. Comput. Harmonic Anal., 25(2008), 148-167. MR2436767 (2009k:47135)

[22] Q. Sun, Wiener's lemma for infinite matrices, Trans. Amer. Math. Soc., 359(2007), 30993123. MR2299448 (2008e:47036)

[23] Q. Sun, Nonuniform average sampling and reconstruction of signals with finite rate of innovation, SIAM J. Math. Anal., 38(2006/07), 1389-1422. MR.2286012(2008b:94049)

[24] W. C. Sun and X. W. Zhou. Reconstruction of functions in spline subspaces from local averages, Proc. Amer. Math. Soc., 131(2003), 2561-2571. MR.1974656 (2004f:42057)

[25] M. Unser, Sampling - 50 years after Shannon, Proc. IEEE, 88(2000), 569-587.

[26] G. Wahba, Spline Models for Observational Data, CBMS-NSF Regional Conf. Ser. Appl. Math. 59, SIAM, Philadelphia, 1990. MR1045442 (91g:62028)

[27] G. G. Walter, A sampling theorem for wavelet subspaces, IEEE Trans. Inform. Theory, 38(1992), 881-884. MR1162226 (93e:94006)

[28] J. Xian and S. Li, Sampling set conditions in weighted multiply generated shiftinvariant spaces and their applications, Appl. Comput. Harmon. Anal., 23(2007), 171-180. MR2344609(2008h:94034) 
[29] J. Xian and W. Lin, Sampling and reconstruction in time-warped spaces and their applications, Appl. Math. Comput., 157(2004), 153-173. MR2085530(2005e:42130)

[30] J. Xian, Error estimates from noise samples for iterative algorithm in shift-invariant signal spaces, Abstract and Applied Analysis, 2010, 1-9. MR2737926 (2012e:94053)

Department of Mathematics, University of Central Florida, Orlando, Florida 32816

E-mail address: zuhair.nashed@ucf.edu

Department of Mathematics, University of Central Florida, Orlando, Florida 32816

E-mail address: qiyu.sun@ucf.edu

Guangdong Province Key Laboratory of Computational Science and Department of Mathematics, Sun Yat-sen University, Guangzhou, 510275, People's Republic of China

E-mail address: xianjun@mail.sysu.edu.cn 